(1)

CrossMark

\title{
The CICERO (Collaboration In COPD ExaceRbatiOns) Clinical Research Collaboration
}

\author{
Wim Janssens (10 ${ }^{1}$ and Mona Bafadhel ${ }^{2}$, Chairs of the CICERO Clinical Research \\ Collaboration ${ }^{3}$
}

Affiliations: ${ }^{1}$ Clinical Dept of Respiratory Diseases, UZ Leuven, BREATHE department CHROMETA, KU Leuven, Leuven, Belgium. ${ }^{2}$ Respiratory Medicine Unit, Nuffield Dept of Medicine, University of Oxford, Oxford, UK. ${ }^{3} \mathrm{~A}$ list of CICERO Clinical Research Collaboration members can be found in the acknowledgements section.

Correspondence: Mona Bafadhel, Nuffield Dept of Medicine, University of Oxford, NDM Research Building, Old Road Campus, Oxford, OX3 7FZ, UK. E-mail: mona.bafadhellandm.ox.ac.uk

@ERSpublications

CICERO is a new Clinical Research Collaboration (CRC), studying severe exacerbations of COPD http://bit.ly/30K2BDi

Cite this article as: Janssens W, Bafadhel M. The CICERO (Collaboration In COPD ExaceRbatiOns) Clinical Research Collaboration. Eur Respir J 2020; 55: 2000079 [https://doi.org/10.1183/13993003.000792020].

Marcus Tullius Cicero was a famous Roman (b. 106 BC) with enterprise in politics, philosophy and oration. Cicero was recognised as a forefather of modernising Latin and Latin prose and for providing the building blocks of many European languages. His most famous of works De Officis was the second book to be printed in Europe after the Gutenberg Bible (c1455), establishing his relevance to both the European and global community. One of Cicero's most famous quotes is “Quousque tandem abutere, patientia nostra" said to Lucius Sergius Catilina (b. 108 BC) upon exposure of the plot to overthrow Rome (c. 63 $\mathrm{BC}$ ). "When, do you mean to stop abusing our patience", the translation of Cicero's most famous quote, is highly relevant when pointing to the necessity and urgency of this new Clinical Research Collaboration (CRC).

Despite almost 23 million patients living with COPD in Europe today, morbidity and mortality associated with the disease remains unacceptably high [1]. Outcomes following an exacerbation of COPD have not changed for many decades and, in Europe, COPD accounts for more than 1.1 million hospital admissions annually, with an estimated mortality of more than $10 \%$ within 3 months post admission [2]. The majority of current and guideline approved interventions have focused on the prevention of exacerbations, but the acute crisis itself [3] has been left understudied. In the case of a severe event, often leading to hospital admission, there is real deficiency of data. Healthcare practitioners and our patients struggle to define the event, there are no recommendations on what to do with regard to characterising or monitoring when it occurs, and we are left with recommendations of acute treatments that have not changed for approximately four decades. The need is thus high to redefine priorities and to overcome the main barriers to fighting against COPD [4]. Supported by European Respiratory Society assemblies 1, 5 and 6, a new CRC was born focusing on severe COPD exacerbations [5] and asking, as Cicero did, "when will our patience for drastic improvements be stopped?" 
TABLE 1 CICERO (Collaboration In COPD ExaceRbatiOns) objectives

Objective 1 Establish European CICERO centres to take part in longitudinal data collection cohort CATALINA study

Objective 2 European CICERO expert consultation survey of what/when to measure during and after hospitalised COPD exacerbation event

Objective 3 Establish standardisation of data collection, processing and storage of all samples

Objective 4 Set up CICERO resources for members, partners, patients and the public

Objective 5 Create change in clinical practice via European Respiratory Society approved task forces

Objective 6 CICERO extension with further funding for interventional studies

The Collaboration In COPD ExaceRbatiOns (CICERO) CRC is an ambitious project with the overarching aim to understand and improve outcomes in severe COPD exacerbations across Europe. Firstly, we will establish a pan-European multicentre network, developing collaborations with academic and industry partners, set out to achieve a set of objectives over the first cycle of the CRC (table 1). In the next 3 years, CICERO will develop a network of expert centres, which can operate with standardised protocols and collect clinical, functional and imaging data alongside repeated bio-sampling during and after hospital admission for an exacerbation of COPD. Secondly, embedded within this, is the CATALINA study (figure 1), a prospective observational cohort study designed to recruit 2000 patients hospitalised with acute COPD exacerbations with longitudinal follow-up. Mandatory data collection in the CATALINA study will be defined by a Delphi approach to reach a Europe-wide consensus on the most important process and outcome parameters to be measured. In parallel, samples from sputum and blood will be collected at fixed time-points and locally stored for future analysis. The potential of this unique data collection and biobank will be vast. We believe our approach will help us in defining phenotypes of exacerbations by the identification of clinical fingerprints, specific biomarkers and corresponding interventions, but it will also validate process and outcomes parameters that are key for individual patient and population follow-up. Most importantly, the CATALINA study as part of CICERO will serve as an exploratory dataset to design future interventions studies for specific patient subgroups. CICERO centres will be given the tools to operate as an accessible platform ready to execute hypothesis testing trials with a standardised approach. Recruitment of CICERO centres will be made in two phases, with phase 1 complete so far. As such, CICERO will not only nourish our need for better scientific insight in the heterogeneity of the problem, it will also translate our findings directly into clinical practice.

And finally, CICERO also has the ambition to create awareness on the appropriate management of exacerbations, since multidisciplinary interventions, individualised approaches and patient perspectives are

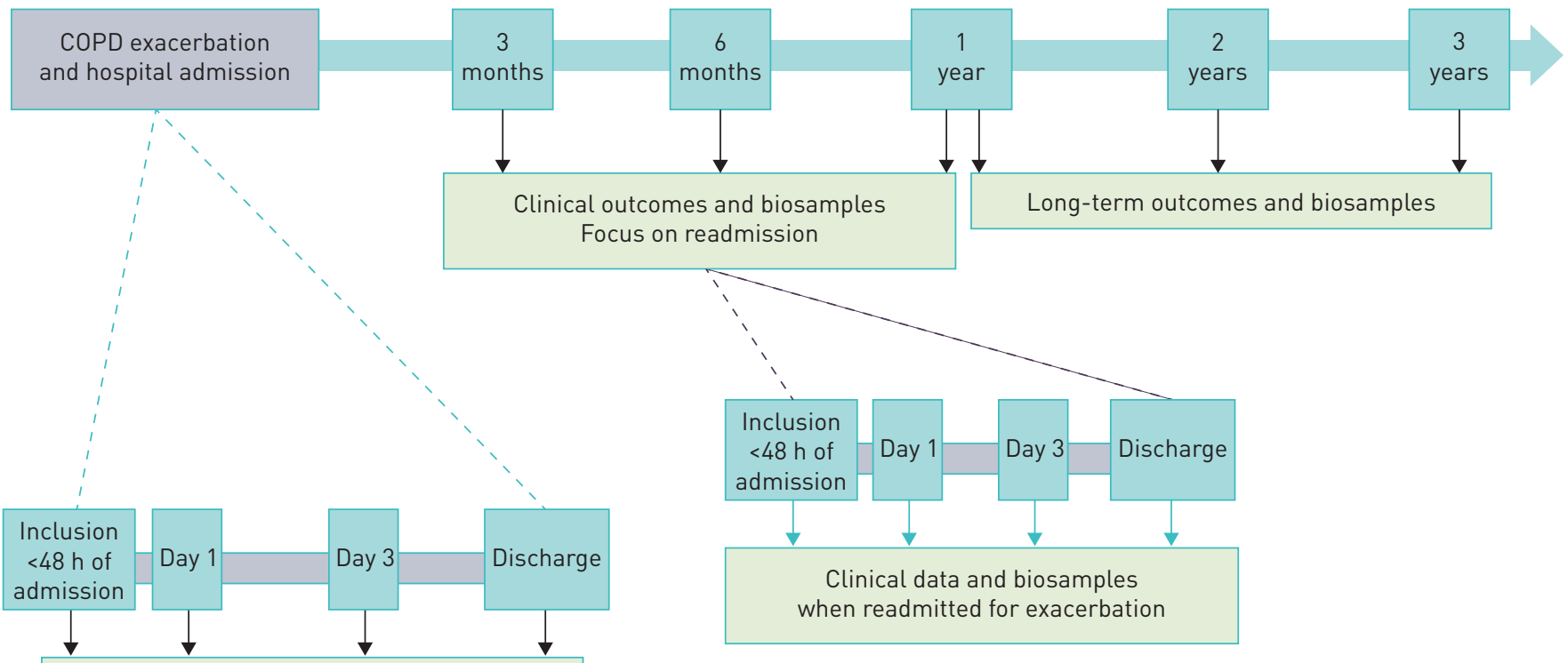

Extensive data collection and biosamples

FIGURE 1 Proposed study design of CATALINA. 
often neglected in daily practice. In collaboration with the European Lung Foundation and patient representatives, we will develop access to resources necessary for the management of COPD exacerbations, organising educational seminars for healthcare professionals and the deployment of several advocacy initiatives to all stakeholders, including patient and public representatives.

In summary, CICERO will create a collaborative enterprise between partners operating at the frontline of COPD research. By cross-fertilisation, open communication and broad access to a unique dataset, CICERO will have the power to shape this blank field. Expert centres capable of following our approach as well as industrial partners eager for innovation, are thus welcome to join. In following Marcus Tullius Cicero, we propose "docere, delectare et movere": to prove our case, make patients smile and take further action.

Acknowledgements: This article was written on behalf of the CICERO Clinical Research Collaboration members. Founding members: Pierre Regis-Burgel, France; Marco Contoli, Italy; Lowie Van Fleteren, Sweden; Arturo Huerta Garcia, Spain; Frits Franssen, The Netherlands; Neil Greening, UK; Timm Greulich, Germany; Jennifer Quint, UK; Henrik Watz, Germany. Young investigators: Sanjay Ramakrishnan, Australia; Iwein Gyselinck, Belgium; Andreas Halner, UK; Kristina Vermeersch, Belgium. Advisory panel: Richard Albert, USA; Christine Jenkins, Australia; Claus Vogelmeier, Germany. Patient and European Lung Foundation representatives: Philip Collis; Hilma Bolsman; Courtney Coleman. Supporting industry partners: AstraZeneca; GlaxoSmithKline; Roche.

Conflict of interest: W. Janssens reports research funding and personal fees for lectures and consultancy from Boehringer Ingelheim, AstraZeneca, Novartis, Chiesi and GlaxoSmithKline, and is a founder of ArtIQ, outside the submitted work. M. Bafadhel reports grants from AZ, personal fees for consultancy and advisory board work and non-financial support (travel to meetings) from AZ, Chiesi and GSK, and is scientific advisor to and minor shareholder in AlbusHealth, outside the submitted work.

\section{References}

1 Gibson GJ, Loddenkemper R, Lundback B, et al. Respiratory health and disease in Europe: the new European Lung White Book. Eur Respir J 2013; 42: 559-563.

2 Hartl S, Lopez-Campos JL, Pozo-Rodriguez F, et al. Risk of death and readmission of hospital-admitted COPD exacerbations: European COPD Audit. Eur Respir J 2016; 47: 113-121.

3 Bafadhel M, Criner G, Dransfield MT, et al. Exacerbations of chronic obstructive pulmonary disease: time to rename. Lancet Respir Med 2020; 8: 133-135.

4 Dransfield M, Stolz D, Kleinert S. Towards eradication of chronic obstructive pulmonary disease: a Lancet Commission. Lancet 2019; 393: 1786-1788.

5 Brightling C, Genton C, Bill W, et al. ERS Clinical Research Collaborations: underpinning research excellence. Eur Respir J 2018; 52: 1801534. 